\title{
Selection of the Location of Point Excitation Force of Plate Measurement Model about Decoupling Coating
}

\author{
LIU Gui-feng \\ Training Department, Naval University \\ of Engineering \\ Hubei Wuhan, China, 430033
}

\author{
HE Shi-ping \\ College of Power, Naval University of \\ Engineering \\ Hubei Wuhan, China, 430033
}

\author{
Shi Lianhui \\ College of Power, Naval University of \\ Engineering \\ Hubei Wuhan, China, 430033
}

\begin{abstract}
The location of point excitation force is an important factor to influence sound radiation of underwater structure. A vibroacoustic model of a simply supported rectangular plate covered by decoupling coating is presented. The influences of the locations and the number of point excitation force to sound radiation power insertion loss are analyzed. The results show that 1) The location of point excitation force has much influence to sound radiation power insertion loss; 2 ) The average sound radiation power insertion loss is closer to convergence as the number of point excitation force becomes larger; 3) When The location of point excitation force is closer to the corner of plate, sound radiation power insertion loss compares well with that of multi-point average. When plate model is used to measure the performance of noise reduction of decoupling coating, the location of point excitation force should be located of the position from a corner of the tested plate so that as many plate modes as possible are excited.
\end{abstract}

Keywords- decoupling coating; insertion loss; location of point excitation force

\section{INTRODUCTION}

Decoupling coating is an important measurement to reduce sound radiation of underwater structure[1-3]. It consists of flexible viscous-elastic material and is coated on the outside of underwater structure. The aim of the treatment is to isolate the fluid from the vibration of the structures, so as to reduce radiation noise.

The noise reduction performance of decoupling coating is often tested by underwater acoustic tube and big sample model in lake. The measurement of underwater sound transmission loss is one of the most common means to test the noise reduction performance of decoupling coating. Sound transmission loss is defined as the decibels of the reciprocal of transmission coefficient, which is the insertion loss, defined by national standard GB/T14369-93 "Acoucstics-Measurement method of insertion loss and echo reduction for the underwater acoustical material properties"[4]. The standard specified that the test condition is that the two sides of the underwater sound materials (including Decoupling coating) are surrounded by water. The measurement methods are Pulse tube method and free-field method. Many authors have carried out the study of the noise reduction performance of decoupling coating around sound transmission loss[5,6]. In the test of sound transmission loss, structure-borne sound travels by longitudinal wave. However, the actual structure-borne sound travels mainly by flexural wave. Meng Tao, Wei-Lin Tang and other scholars indicated that the usual means of sound transmission loss can't directly reflect the noise reduction performance of decoupling coating[1].

One way to test the noise reduction performance of decoupling coating is to test the radiated sound power before and after covering decoupling coating on the cylindrical shell scale model in lake or anechoic tank, to obtain the insertion loss of radiated sound power [7,8]. However, scale model can hardly imitate the thickness of shell and acoustics coating; it is more suitable for theoretical study or characteristic analysis of decoupling coating, rather than the evaluation of the noise reduction performance of decoupling coating.

The other way to evaluate the noise reduction performance of decoupling coating in present is to test the radiated sound power before and after covering decoupling coating on the plate model in anechoic tank, so as to obtain the insertion loss of radiated sound power. The thickness of plate model is the same as that before covering the decoupling coating. This test method has a problem that the mode varies when the location of point excitation force changes, which causes the change of the radiated sound power.

The paper presents a model to simulate the vibration and sound radiation on the simply supported rectangular plate. On that basis, the influence of the location of point excitation force on the insertion loss of radiated sound power is analyzed, and suggested that the selection on the location of point excitation force.

\section{THEORECTICAL MODEL}

\section{A. Statement of the problem}

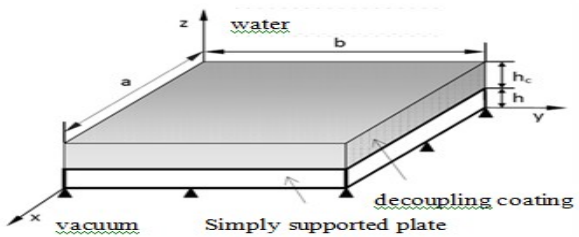

Fig.1 Schematic representation of the simply supported plate covered with a decoupling coating

The structure is assumed to be a simply supported rectangular plate inserted in an infinite rigid baffle that separates vaccum on one side $(\mathrm{z}<0)$ from water on the other side $(\mathrm{z}>0)$ (Fig. 1). The plate is assumed to conform to the Love- 
Kirchhoff theory that considers only bending deformation, while the coating is described with the fluid-like model. The plate is excited by a harmonic point force.

\section{B. Description of Equation of Motion}

The equation of motion of the plate, expressed in the frequency domain, is

$$
\tilde{D} \nabla^{4} \tilde{w}_{1}(Q, \omega)-\rho_{S} h \omega^{2} \tilde{w}_{1}(Q, \omega)=\tilde{f}(Q, \omega)-\tilde{\sigma}(Q, \omega)
$$

where $\mathrm{w}$ is the angular frequency, $Q$ is a point on the plate, $\tilde{D}$ is the complex bending stiffness of the plate: $\tilde{D}=\tilde{E} h^{3} / 12\left(1-v^{2}\right), \rho_{s}, h, v$ are respectively the density, the thickness, and the Poisson's ratio of the plate. $\tilde{E}$ is the complex Young's modulus, defined by $\tilde{E}=E(1+j \eta), E$ is the real part of Young's modulus, and $\eta$ is the structural loss factor, $\nabla^{4}$ is a partial differential operator: $\nabla^{4}=\frac{\partial^{4}}{\partial x^{4}}+2 \frac{\partial^{4}}{x^{2} y^{2}}+\frac{\partial^{4}}{\partial y^{4}} \cdot \tilde{f}(Q, \omega)$ is the external transverse force per unit area applied to the base plate and $\tilde{\sigma}(Q, \omega)$ is the normal stress in the z-direction applied by the coating on the plate. $\tilde{w}_{1}(Q, \omega)$ is the transverse displacement of the plate.

The transverse displacement of the plate is written in terms of the in-vacuo modes of the plate, which satisfy the simply supported boundary condition (displacement and flexural moment equal to zero):

$$
\begin{gathered}
\tilde{w}_{1}(Q, \omega)=\sum_{m=1}^{\infty} \sum_{n=1}^{\infty} \tilde{a}_{m n}(\omega) W_{m n}(Q) \\
\text { where } W_{m n}(Q)=\sin \left(m \pi \frac{x}{a}\right) \sin \left(m \pi \frac{y}{b}\right) .
\end{gathered}
$$

Generally, the dynamic stiffness of decoupling coating is small, and shear waves have little influence on the vibration and underwater sound radiation of the plate. Here, it is assumed that shear waves don't affect the motion of the plate and decoupling coating. Then, the decoupling coating is assumed as fluid-like media. Applying transfer matrix method, the relation between normal stress $\tilde{\sigma}(Q, \omega)$ in the z-direction applied by the coating on the plate, the acoustic pressure $\tilde{p}(Q)$ of the outer surface of decoupling coating and the transverse displacement $\tilde{w}_{1}(Q, \omega)$ of the plate, the transverse displacement $\tilde{w}_{2}(Q, \omega)$ of the outer surface of decoupling coating could be expressed as

$$
\left(\begin{array}{l}
\tilde{\sigma}(Q) \\
\tilde{p}(Q)
\end{array}\right)=\frac{\rho c \omega}{\sin \left(k h_{c}\right)}\left[\begin{array}{cc}
\cos \left(k h_{c}\right) & -1 \\
1 & \cos \left(k h_{c}\right)
\end{array}\right]\left(\begin{array}{c}
\tilde{w}_{1}(Q) \\
\tilde{w}_{2}(Q)
\end{array}\right)
$$

where $\rho, h_{c}, c$ are respectively the density, the thickness, and the sound velocity of the decoupling coating. $k$ is the wave

number of longitudinal wave of the decoupling coating $k=\omega / c$.
Assuming the complex Young's modulus of decoupling coating is $\tilde{E}_{c}=E_{c}\left(1+j \eta_{c}\right), E_{c}$ is the real part of Young's modulus, and $\eta_{c}$ is the loss factor, the sound velocity of longitudinal wave can be expressed as

$$
c=\sqrt{\tilde{E}_{c} / \rho}
$$

The acoustic pressure in the fluid is governed by the Helmholtz equation,

$$
\nabla^{2} \tilde{p}(M)+k_{0}^{2} \tilde{p}(M)=0 \quad\left(k_{0}=\omega / c_{0}\right)
$$

where $M$ is a point in the fluid space, $c_{0}$ is the velocity of sound in the fluid.

The equation of continuity of the structural and acoustic normal acceleration on the outer surface of the decoupling material is

$$
\frac{\partial \tilde{p}(M)}{\partial z}=\rho_{0} \omega^{2} \tilde{w}_{2}(Q)
$$

where $\rho_{0}$ is the density of the fluid.

The acoustic pressure on the surface of the coating is expressed as the Rayleigh integral[10],

$$
\tilde{p}(Q)=-\iint\left[\frac{\partial \tilde{p}(M)}{\partial n_{M}} G(Q, M)\right] d s_{M}
$$

Where $S$ is the surface of the decoupling coating, points $Q$ and $M$ are on the surface of the decoupling coating, $G(Q, M)$ is acoustic Green's function,

$$
G(Q, M)=G\left(x, y, x^{\prime}, y^{\prime}\right)=\frac{e^{-j k_{0} \sqrt{\left(x-x^{\prime}\right)^{2}+\left(y-y^{\prime}\right)^{2}}}}{2 \pi \sqrt{\left(x-x^{\prime}\right)^{2}+\left(y-y^{\prime}\right)^{2}}}
$$

The transverse displacement of the outer surface of the decoupling coating is written in terms of the in-vacuo modes of the plate:

$$
\tilde{w}_{2}(Q, \omega)=\sum_{m=1}^{\infty} \sum_{n=1}^{\infty} \tilde{b}_{m n}(\omega) W_{m n}(Q)
$$

Using Eq.(3),(6) and (9), Eq.(7) becomes

$$
\begin{aligned}
& -\iint_{s}\left[\rho_{0} \omega^{2} \tilde{w}_{2}(Q) G(Q, M)\right] d s_{M} \\
& =\frac{\rho c \omega}{\sin \left(k h_{c}\right)}\left[\tilde{w}_{1}(Q)-\cos \left(k h_{c}\right) \tilde{w}_{2}(Q)\right]
\end{aligned}
$$

Using the orthogonal properties of the natural modes, Equations (1) and (10) are two systems of coupled equations in terms of the modal unknows of the problems: $\tilde{a}_{m n}$ and $\tilde{b}_{m n}$ $\forall(m, n) \in[1, \cdots N]^{2}$, and be written in 


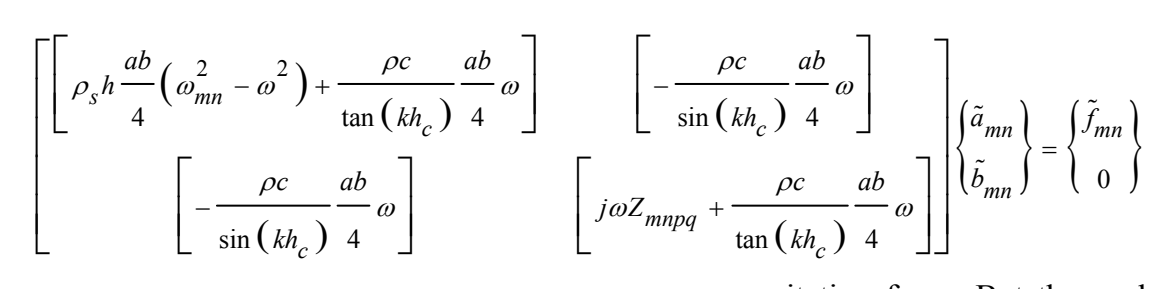

where $Z_{\text {mnpq }}$ are the coefficients of the radiation impedance matrix defined by:

$$
Z_{m n p q}=j \rho_{0} \omega \iint_{S} \iint_{S} W_{p q}(M) G(Q, M) W_{m n}(Q) d s_{M} d s_{Q}
$$

$\omega_{m n}$ is the in-vacuo natural angular frequencies of the plate:

$$
\omega_{m n}=\sqrt{\frac{D}{\rho_{s} h}}\left(\left(\frac{m \pi}{a}\right)^{2}+\left(\frac{n \pi}{b}\right)^{2}\right)
$$

$\tilde{f}_{m n}$ is the generalized vector of forces:

$$
\tilde{f}_{m n}=F_{0} \sin \left(m \pi \frac{x_{0}}{a}\right) \sin \left(n \pi \frac{y_{0}}{b}\right)
$$

where $F_{0}$ is the magnitude of the force, and $x_{0}$ and $y_{0}$ are the coordinates of the excitation point.

To solve the problem, it is needed to truncate the infinite series (m,n,p,q) to a finite order N. Equation (11) becomes a matrix system with dimensions $2 N^{2} \times 2 N^{2}$.The unknown coefficients $\tilde{a}_{m n}$ and $\tilde{b}_{m n}$ can be calculated, which allows vibration velocity and sound radiation power to be calculated.

The insertion loss of sound radiated power is defined as:

$$
I L_{w}=10 \log \left(W_{p} / W_{c}\right)
$$

where $w_{p}, w_{c}$ are respectively the sound radiated power of the plate with and without the decoupling coating

\section{THE EFFECT OF THE LOCATION OF EXCITATION POINTS ON SOUND RADIATION POWER INSERTION LOSS}

One side of the simply supported rectangular plate is air, and the other is immersed in water with $\left(\rho_{0}=1000 \mathrm{~kg} / \mathrm{m}^{3}\right.$, $c_{0}=1500 \mathrm{~m} / \mathrm{s}$ ), the surface of the rectangular plate adjacent to the water medium is all covered by decoupling coating. The length, width and thickness of the substrate plate are respectively $a=750 \mathrm{~mm}, b=1000 \mathrm{~mm}, h=20 \mathrm{~mm}$. The density of the substrate plate is $\rho_{\mathrm{s}}=7800 \mathrm{~kg} / \mathrm{m}^{3}$, the Young's modulus is $E=2 \times 10^{11}$ $\mathrm{N} / \mathrm{m}^{2}$, Poisson's ratio $v=0.3$. The thickness of decoupling coating is $h_{c}=30 \mathrm{~mm}$, the complex Young's modulus is $E_{c}=5 \times$ $10^{6}(1+0.1 i) \mathrm{N} / \mathrm{m}^{2}$, and the density $\rho=1100 \mathrm{~kg} / \mathrm{m}^{3}$.

The Fig. 2 gives the curves that the sound radiation power insertion loss varied with frequency at the two different location of point excitation force (the center $\left(\mathrm{x}_{0}=a / 2, \mathrm{y}_{0}=b / 2\right)$ and the corner $\left(\mathrm{x}_{0}=a / 8, \mathrm{y}_{0}=b / 8\right)$ of the plate). The abscissa is logarithm of the frequency. The results show that the whole trend of the sound radiation power insertion loss varied with frequency are consistent on two different positions of point excitation force. But the peak number of the sound radiation power insertion loss is significantly less than the number at the corner of the substrate plate when the location of point excitation force is the center of the substrate plate. The reason is that many of the modes can not be excited at the center of the substrate.

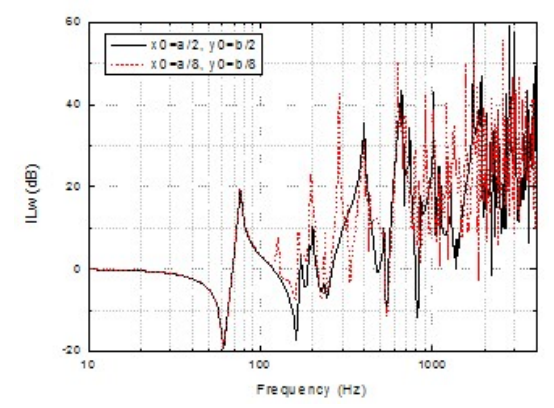

Fig. 2 Sound radiated power insertion loss; solid line: $(\mathrm{x} 0=\mathrm{a} / 2, \mathrm{y} 0=\mathrm{b} / 2)$; dash line: $(\mathrm{x} 0=\mathrm{a} / 8, \mathrm{y} 0=\mathrm{b} / 8)$

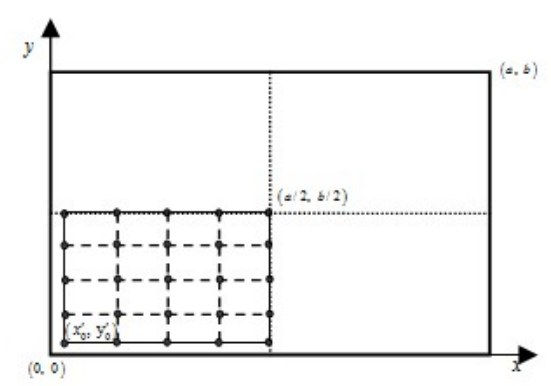

Fig.3 Selection of the location of point excitation force

Substituting the coordinate of the central excitation point $\left(\mathrm{x}_{0}=a / 2, \mathrm{y}_{0}=b / 2\right)$ into the formula(14), derived that:

$$
f_{m n}=F_{0} \sin \left(\frac{m \pi}{2}\right) \sin \left(\frac{n \pi}{2}\right)
$$

When $\mathrm{m}$ or $\mathrm{n}$ is even, $f_{m n}=0$, and the corresponding mode can't be excited because the excitation point happens to be at the position of the mode's node. Only when $\mathrm{m}$ and $\mathrm{n}$ is all odd, the corresponding mode will be excited. Supposing that the maximum value of $\mathrm{m}$ and $\mathrm{n}$ is 10 , then only 25 modes can be excited in all 100 ones.

Substituting the coordinate of the corner excitation point $\left(\mathrm{x}_{0}=a / 8, \mathrm{y}_{0}=b / 8\right)$ into the formula(14), derived that:

$$
f_{m n}=F_{0} \sin \left(\frac{m \pi}{8}\right) \sin \left(\frac{n \pi}{8}\right)
$$

Now, when $\mathrm{m}$ or $\mathrm{n}$ is integer multiple of $8, f_{m n}=0$, and the corresponding mode can't be excited. Similarly, supposing that 
the maximum value of $m$ and $n$ is 10 , then most of the 100 modes can be excited.

From the results and analysis of Fig.2, the sound radiation power insertion loss are of significant difference, because the modes on different excitation points are various. Then, how to select the position of excitation points when using the plate model to evaluate the noise reduction performance of decoupling coating?

A simple solution is to select a sufficient number of excitation points, and all excitation forces are not relevant, then selecting the average radiated sound power value of multiple excitation points.Due to the symmetry of the plate, the position of excitation point can be selected in the quarter area of the plate. Supposed that a rectangular area which diagonal coordinates are $\left(x_{0}^{\prime}, y_{0}^{\prime}\right)$ and $(a / 2, b / 2)$ is equally divided into $\mathrm{N}_{1}$ sections in the direction of abscissa and ordinate, then the equal division points are selected as the excitation points. Take $x_{0}^{\prime}=a / 8, y_{0}^{\prime}=b / 8, \mathrm{~N}_{1}=5$. Fig. 4 compares the two curves of the average radiation sound power insertion loss varied with frequency on 25 excitation points and 24 ones. The results show that two average radiation sound power insertion loss are basically the same.

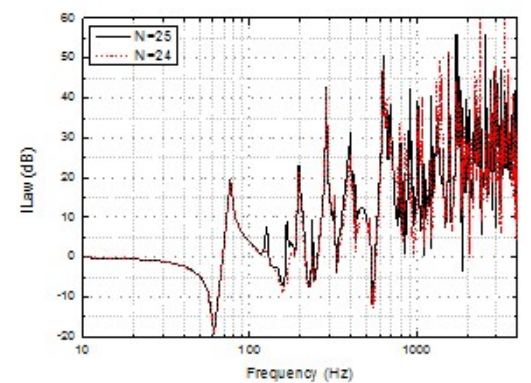

Fig.4 Average sound radiated power insertion loss; solid line: $\mathrm{N}=25$; dash line: $\mathrm{N}=24$

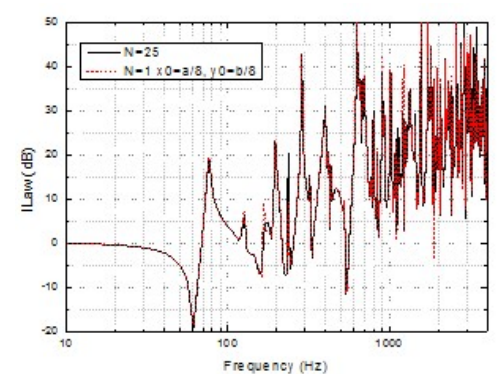

Fig.5 Average sound radiated power insertion loss; solid line: $\mathrm{N}=25$; dash line: $\mathrm{N}=1 \quad(\mathrm{x} 0=\mathrm{a} / 8, \mathrm{y} 0=\mathrm{b} / 8)$

However, in the actual plate model test system, it's a huge work to test multiple excitation point. If we can find a point that consistent with the average radiation sound power insertion loss of multiple excitation point, the test will be easier. Received from formula (14), excitation point the more closer to the corner of the plate, the more modes number will be excited.
Fig. 5 compares the radiation sound power insertion loss on the excitation point $\left(\mathrm{x}_{0}=a / 8, \mathrm{y}_{0}=b / 8\right)$ and the average value of all 25 excitation points. The fig. 5 concluded that the two results are unanimous. i.e. The corner of the plate is very suitable for the location of point excitation force.

\section{CONCLUSION}

This thesis builds a vibration and sound radiation model of the simply supported rectangular plate which is water-loaded on one side and air backed on the other under the effect of point excitation force. The influence of the number and location of excitation points on the sound radiation power insertion loss is analyzed. The research shows that:

1) The location of point excitation force has great influence on the sound radiation power insertion loss.

2) the more number of point excitation forces, the results of the sound radiation power insertion loss more closer to convergence.

3) the radiation sound power insertion loss that the location of point excitation force is the corner of the plate is basically consistent with the average value of many excitation points

The excitation point selection is more appropriate to the corner of the plate, when using the plate model to evaluate the noise reduction performance of decoupling coating.

\section{REFERENCES}

[1] Tao meng, Tang Weilin, Fan Jun. "Mechanism analysis of noise reduction by compliant decoupling layers," Journal of Ship Mechanics, Vol.14(4), pp. 220-225. In Chinese

[2] Tao meng, Fan Jun, Tang Weilin. "The characteristics of sound radiation from a cylindrical shell coated with multiple compliant layers," ACTA ACUSTICA, 2008,Vol.33(3), pp. 421-429. In Chinese

[3] Miao Xuhong, Wang Renqian, Gu lei. "Numerical analysis on the sound insulation performance of decoupling material," Journal of Ship Mechanics, 2005,Vol.9(5), pp. 125-131. In Chinese

[4] Wang Rongjin, Miao Rongxing. "Acoucstics-Measurement method of insertion loss and echo reduction for the underwater acoustical material properties," GB/T 14369-93. In Chinese

[5] Brekhovshikh. Acoustics of layered media. Springer-Verlag Berlin Heidelberg. 1985.

[6] Panigrahi S N, Jog C S, Munjal M L. "Multi-focus design of underwater noise control lining based on finite element analysis". Applied acoustics, 2008,69, pp. 1141-1153.

[7] Yao Xiongliang, Zhang Aman, Qian Dejin, Pang Fuzhen. "On effect of laying regious of uncoupled anechoic material on sound radiation of double cylindrical shell," Journal of naval university of engineering, 2008, 20(2):33-37. In Chinese

[8] Yao Xiongliang, Zhang $\mathrm{Xu}$, Qian Dejin, Zhang Aman. "Experiment research of acoustics performance of separate-sound and decoupled material," Transducer and microsystem technologies, 2008, 27(7):31-33. In Chinese

[9] S.Nikiforov, translated from Russian by Alexander Katalov and Dimitri Donskoy. Acoustic design of naval structures. USA, Naval Surface Warfare Center, 2005.

[10] He ZuoYong. Structural Vibration and Sound radiation. Harbin Engineering University. 2001 